\title{
P12 Proliferation and Procoagulant Activity of Vascular Smooth Muscle Cells from Thoracic and Abdominal Aortic Aneurysms
}

\author{
Melusine Didelot ${ }^{1}$, Jeremy Lagrange ${ }^{1}$, Jean-Baptiste Michel$^{2}$, Patrick Lacolley ${ }^{1}$, Veronique Regnault ${ }^{1}$
}

${ }^{1}$ Inserm U1116, Nancy, France

${ }^{2}$ Inserm U1148, Paris, France

\begin{abstract}
In contrast to abdominal aortic aneurysm (AAA), aneurysms of the thoracic ascending aorta (TAA) are not associated with a mural thrombus. Mechanistic understanding of the involvement of vascular smooth muscle cells (VSMCs) in thrombus formation is lacking. We aim to determine (i) whether thrombin generation at the surface of VSMCs is increased in AAA, (ii) the contribution of the avb3 integrin as a receptor for prothrombin, (iii) the implication of protease activated receptor (PAR) activation in the thrombin generation, and (iv) the capacity of thrombin to induce VSMC proliferation. Primary cultures of VSMCs were prepared from human biopsies of TAA, AAA and healthy aorta ( $n=11$ /group). In the presence of prothrombin deficient plasma, similar low levels of thrombin were formed on the 3 types of VSMCs. In the presence of healthy plasma, thrombin generation was higher on VSMCs from AAA and lower on VSMCs from TAA compared with controls. Incubation of VSMCs with a RGT peptide inhibiting the b3 signaling reduced the amount of thrombin in the 3 groups. Incubation of healthy VSMCs with a selective PAR-1 antagonist decreased thrombin generation. This inhibition was more pronounced for AAA (36\%) than from TAA (17\%). VSMCs from AAA were more sensitive to thrombin-induced proliferation than VSMCs from TAA. The prothrombotic phenotype and proliferation of VSMCs from AAA compared to TAA is mediated partly by PAR-1. This argues for a contribution of VSMC in the occurrence of thrombotic events in AAA. Thus, inhibition of PAR signaling represents a new target in this complex disease.
\end{abstract}

(c) 2019 Association for Research into Arterial Structure and Physiology. Publishing services by Atlantis Press International B.V. This is an open access article distributed under the CC BY-NC 4.0 license (http://creativecommons.org/licenses/by-nc/4.0/). 